\title{
Germanica
}

\section{Georg von der Vrings Die Spur im Hafen. Mord und Detektion im Dritten Reich}

Die Spur im Hafen de Georg von der Vring. Meurtres et détection sous le

Troisième Reich

Georg von der Vring's Die Spur im Hafen. Murder and Detection in the Third Reich

Elena Giovannini

\section{OpenEdition}

\section{Journals}

Édition électronique

URL : http://journals.openedition.org/germanica/3175

DOI : 10.4000/germanica.3175

ISSN : 2107-0784

\section{Éditeur}

Université de Lille

\section{Édition imprimée}

Date de publication : 30 septembre 2016

Pagination : 43-51

ISBN : 9782913857377

ISSN : 0984-2632

\section{Référence électronique}

Elena Giovannini, «Georg von der Vrings Die Spur im Hafen. Mord und Detektion im Dritten Reich », Germanica [Online], 58 | 2016, Online erschienen am: 30 September 2018, abgerufen am 06 Oktober 2020. URL : http://journals.openedition.org/germanica/3175;DOI : https://doi.org/10.4000/ germanica.3175 


\section{Le roman policier sous le III' Reich}





\title{
Georg von der Vrings Die Spur im Hafen. Mord und Detektion im Dritten Reich
}

\author{
Elena GIOVANNINI \\ Università degli Studi di Bologna
}

Der Vorbehalt gegen die literarische Darstellung von Kriminaltaten war unter den Spitzen der NS-Diktatur weitverbreitet. Kriminalromane wurden im Dritten Reich oft als suspekt und destabilisierend angesehen, denn ihnen wurde vorgeworfen, zu Verbrechen anzustiften, der deutschen Tradition und ihren Werten nicht zu entsprechen, den Vorrang des Individuums vor der Gemeinschaft zu betonen und für die angloamerikanische Kultur zu werben. Trotzdem war die Kriminalliteratur in Hitlers Deutschland sehr populär und spielte dank den zahlreichen Veröffentlichungen und den hohen Auflagen der Publikationen sowohl in Buchform als auch in wöchentlichen Heftromanen eine wichtige Rolle in der Buchbranche ${ }^{1}$.

1. - $\mathrm{Zu}$ den Gegnern dieser Gattung und ihren Stellungsnahmen siehe u.a. Irmtraud Götz von Ohlenhusen, ,Mord verjährt nicht. Krimis als historische Quelle (1900-1945)“, S. 124, in: Barbara Korte und Sylvia Paletschek (Hrsg.), Geschichte im Krimi. Beiträge aus den Kulturwissenschaften, Köln u.a., Böhlau, 2009, S. 105130; Carsten Würmann, „Zum Kriminalroman im Nationalsozialismus“, S. 158, in: Bruno Franceschini und Carsten Würmann (Hrsg.), Verbrechen als Passion. Neue Untersuchungen zum Kriminalgenre, Berlin, Weidler, 2004, S. 143-188; Walter T. Rix, ,Romanzo poliziesco e dittatura“, S. 198, in: Renzo Cremante und Lors Rambelli (Hrsg.), La trama del delitto. Teoria e analisi del racconto poliziesco, Parma, Pratiche, 
Die Bürger des Dritten Reiches hatten eine große Unterhaltungslust, die das Regime einerseits befriedigen und andererseits zu volkspädagogischen und propagandistischen Zwecken lenken wollte. Vor allem nach Kriegsbeginn, als das Bedürfnis nach leichten und unterhaltsamen Lektüren deutlich stieg, wirkten die Nationalsozialisten auf die Kriminalliteratur intensiver ein. Bevorzugte Ziele waren die Erziehung zur Verbrechensbekämpfung, der Schutz der Jugend und die Durchsetzung einer ,gesunden' und systemkonformen Unterhaltung. Im Unterschied zur hohen Literatur, zu Film, Rundfunk und Presse fand also die gründlichere Gleichschaltung der Kriminalliteratur erst ab 1939/1940 statt, und trotz der Indizierung wurden weniger drastische Maßnahmen ergriffen ${ }^{2}$.

Unter den 343 Kriminalromanen, die 1936 vor der Durchsetzung der meisten Verbote und Beschlagnahmungen erschienen, fällt Die Spur im Hafen auf, denn dieses Werk erreichte mit mehr als 350.000 Exemplaren die höchste Auflage im Dritten Reich und wurde von der damaligen Kritik sehr positiv aufgenommen ${ }^{3}$. Der Autor - Georg von der Vring wurde in der Weimarer Republik dank seinem, Antikriegsroman' Soldat Suhren (1927) bekannt; in Hitlers Deutschland führte er seine künstlerische Tätigkeit als innerer Emigrant hauptsächlich auf den Gebieten der Lyrik und der Unterhaltungsromane fort. Obwohl von der Vring 1934 sein Lektorat am Südfunk wegen der Verweigerung des Hitlergrußes verlor und sich während der Diktatur für die Parteipolitik nicht interessierte, nahm er 1938, 1940 und 1942 an den ,Weimarer Dichtertreffen' teil,

1980, S. 195-204; Hans Daiber, „Nachahmung der Vorsehung“, S. 424-425, in: Jochen Vogt (Hrsg.), Der Kriminalroman, Bd. II, München, Fink, 1992, S. 421-436. Zahlen und Statistiken über die Verbreitung der Kriminalliteratur im Dritten Reich bieten u.a. Christian Adam, Lesen unter Hitler, Berlin, Galiani, 2010, S. 189; Carsten Würmann, Zwischen Unterhaltung und Propaganda. Das Krimigenre im Dritten Reich, Diss., Berlin, 2013, S. 59-65.

2. - Gemildert wurde die Wirksamkeit der Eingriffe u.a. durch die Meinungsverschiedenheiten unter den dafür zuständigen Parteiführern, den entscheidenden Einfluss des Geschmacks der Leser auf den Buchmarkt und den Prioritätenwechsel im Krieg. Details über die ,Gleichschaltung' der Unterhaltungsund Kriminalliteratur sind in folgenden Beiträgen zu finden: Carsten Würmann, „Deutsche Kommissare ermitteln. Der Kriminalroman im ,Dritten Reich““, S. 220-232, in: Walter Delabar, Horst Denkler und Erhard Schütz (Hrsg.), Banalität mit Stil. Zur Widersprüchlichkeit der Literaturproduktion im Nationalsozialismus, Bern u.a., Peter Lang, 1999, S. 217-240; Ine van Linthout, „,Dichter schreibt Unterhaltungsromane!‘. Der Stellenwert der Unterhaltungsliteratur im ,Dritten Reich““, S. 112-120, in: Carsten Würmann und Asgar Warner (Hrsg.), Im Pausenraum des, Dritten Reiches'. Zur Populärkultur im nationalsozialistischen Deutschlands, Bern u.a., Peter Lang, 2008, S. 111-124; Carsten Würmann, Zwischen Unterhaltung und Propaganda, a.a.O., S. 13$14,101-147$.

3. - Carsten Würmann, Zwischen Unterhaltung und Propaganda, a.a.O., S. 63; Christian Adam, Lesen unter Hitler, a.a.O., S. 191. 
die jährlich von Goebbels und dem Ministerium für Volksaufklärung und Propaganda organisiert wurden. Der Republikaner und Demokrat konnte sich also der nationalsozialistischen Vereinnahmung nicht ganz entziehen ${ }^{4}$.

Die Spur im Hafen, von der Vrings erfolgreichster Text im Dritten Reich, trägt den Untertitel „Roman“, obwohl dieser typische strukturelle und inhaltliche Merkmale des Kriminalromans aufweist. Das Werk handelt von dem Assessor Peter Tewes, der zu dem Tod seines „,verunglückt[en]"5 Onkels Ito Diedrich ermittelt. Nachdem der junge Jurist versteckte Notizen über den früheren Mord an dem Ehepaar Hansen findet, mit dessen Aufklärung sich der tote Verwandte jahrelang heimlich beschäftigte, gewinnt auch Ito Diedrichs Tod verdächtige Züge und stellt sich im Laufe der Erzählung als Kriminaltat heraus, deren Anlass eben in dem Interesse des Onkels am „Fall Hansen“6 liegt. Auf unterschiedlichen Zeitebenen können also zwei Figuren (Peter und der tote Ito Diedrich) auf die Rolle des Ermittlers und drei (der Onkel und das achtzehn Jahre früher ermordete Ehepaar) auf die des Opfers zurückgeführt werden. Was einerseits den toten Ermittler mit dem in der Erzählung tätigen und andererseits beide Opfergruppen verbindet, ist der Raum. Wie in vielen im Dritten Reich verfassten Kriminalromanen ist der Schauplatz der Morde und der Detektion eine ruhige Kleinstadt im Einzugsbereich einer Großstadt. Bei von der Vring handelt es sich um Werderfleht bei Hamburg, in der die Chronotopoi ,Hafen' (wo alle Leichen aufgefunden werden und sich die Ermittlung zum Teil vollzieht) und ,Familienhaus، (Schauplatz von wichtigen Stationen der Detektion, von der Lösung des Falles und von Peter Tewes fiktiver Niederschrift des Romans) eine tragende Rolle spielen, denn ,ils se présentent comme les centres organisateurs des principaux événements contenus dans le sujet du roman [... dans lesquels] le temps acquiert un caractère sensuellement concret"7. Hafen und Familienhäuser gelten also als Verdichtung

4. - Wichtige Hinweise auf das Leben, das Werk und die politische Haltung dieses Schriftstellers sind in folgenden Beiträgen enthalten: Schlossmuseum Jever und Schifffahrtsmuseum Brake (Hrsg.), Georg von der Vring 1889-1968. Eine Ausstellung zum 100. Geburtstag des Dichters und Malers, Jever, Mettcker \& Söhne, 1989, S. 1522; Hans Sarkowicz, Alf Mentzer (Hrsg.), Schriftsteller im Nationalsozialismus. Ein Lexikon, Berlin, Insel, 2011, S. 600-603; Hans J. Fröhlich, „Ein Unsoldat zieht in den Krieg. Hans J. Fröhlich über Georg von der Vring: ,Soldat Suhren“ (1927)“, in: Marcel Reich-Ranicki (Hrsg.), Romane von gestern - heute gelesen, Bd. II, Frankfurt a. M., Fischer, 1989, S. 91-97; Wulf Kirsten, „Georg von der Vring“, in: Bayrische Akademie der schönen Künste. Jahrbuch 13, 1999, 2, S. 608-612.

5. - Georg von der Vring, Die Spur im Hafen. Roman, Gütersloh, Bertelsmann, 1936, S. 7.

6. - Ebd., S. 18.

7. - Mikhail Bakhtine, "Formes du temps et du chronotope dans le roman", S. 391, in: Mikhail Bakhtine, Esthétique et théorie du roman, Paris, Gallimard, 1975, S. 235- 
der Zeitspuren im Raum, denn dort überschneiden und verräumlichen sich die Zeitebenen der Morde (1824 und 1842), der Detektion (1842) und der fiktiven Niederschrift des Romans (1854).

Nicht nur die Modernität der Industriegesellschaft charakterisiert die Stoffe der Unterhaltungs- und Kriminalliteratur des Dritten Reiches, sondern auch die Historisierung des Erzählten ${ }^{8}$, wie im Werk von der Vrings, in dem Morde und Detektion lange vor Hitlers Machtergreifung angesiedelt sind. Weitere kennzeichnende Züge des Kriminalromans zu NS-Zeiten zeigt außerdem die Analyse der Figuren und der Ermittlungspraktiken in Die Spur im Hafen ${ }^{9}$. Die im Dritten Reich überbetonte Männlichkeit ${ }^{10}$ erweist sich, wie in der NS-Kriminalliteratur üblich, bei den wichtigsten Figuren des Romans, d. h. dem Ermittler und den Tätern (dem holländischen Konsul und seinem Komplizen). Ideologische Implikationen haben auch die Triebfeder der Morde: Die Verbrecher töteten zuerst das Ehepaar, weil es mit viel Geld verreiste, dann brachten sie Peter Tewes Onkel aus Selbstschutz um, weil er im ,Fall Hansen' der Wahrheit nahe gekommenen war. Das Streben nach Besitz und Reichtum wird als Beweggrund für fiktive Kriminaltaten im Nationalsozialismus oft geschildert, denn Habgier zeigt sich als Folge kapitalistischer Akkumulation, die die Nationalsozialisten bekämpfen; beide Mordfälle wurzeln außerdem im übersteigerten Individualismus, den das Dritte Reich entwertete.

Die Aufdeckung dieser Untaten, die ,im Rücken der Geschichte“11 liegen, ist das Ziel von von der Vrings Amateurdetektiv, der sich aber nicht nur mit der Wahrheitsfindung, sondern auch mit der Liebe beschäftigt. Im Unterschied zu den Ermittlern der traditionellen Kriminalliteratur, die ,zur Ehelosigkeit [...] verdammt [... und] als ein Neutrum begriffen

398.

8. - Vgl. Walter Delabar, ,NS-Literatur ohne Nationalsozialismus? Thesen zu einem Ausstattungsphänomen in der Unterhaltungsliteratur des ,Dritten Reiches““, S. 163, in: Carsten Würmann und Asgar Warner (Hrsg.), Im Pausenraum des ,Dritten Reiches', a.a.O., S. 161-182.

9. - Die Grundzüge dieser Gattung im NS-Deutschland schildern am detailliertesten Carsten Würmanns Beiträge, und insbesondere „Deutsche Kommissare ermitteln“, a.a.O., S. 237-240.

10. - Sie ist eine Grundeigenschaft von Hitlers ,neuem Deutschland" und wird meistens als ,heldisch', ,einsatzbereit' und ,volksbezogen' gepriesen (vgl. u.a. Erich Weißer, „Die Bedeutung der Dichter für die nationalpolitische Bildung“, in: Zeitschrift für die deutsche Bildung, 1939, 6, S. 256; Edgar Kirsch, „Hans Grimms ,Volk ohne Raum“ als Bildungsroman“, in: Dichtung und Volkstum, 1937, 38, S. 488).

11. - Ernst Bloch, „Philosophische Ansicht des Detektivromans“, S. 327, in: Jochen Vogt (Hrsg.), Der Kriminalroman, Bd. II, a.a.O., S. 322-342. Vgl. auch Tzvetan Todorov, “Typologie des Kriminalromans", S. 209-210, in: Jochen Vogt (Hrsg.), Der Kriminalroman. Poetik - Theorie - Geschichte, München, Fink, 1998, S. 209-215. 
[sind], das sowenig erotisch ist wie unerotisch"12, entwickeln viele fiktive Detektive im Dritten Reich Liebesgefühle. Die Lösung des Falles wird zum Schluss oft durch eine Hochzeit gekrönt, weil sich der Nationalsozialismus auch als versittlichende Instanz versteht. Von der Vring schildert ausführlich die Gefühle und Seelenzustände des jungen Assessors, der sich wie zahlreiche Ermittler in NS-Kriminalromanen in eine emanzipierte Frau verliebt; nach der Aufklärung des Falles wird sie aber zu einer treuen Ehefrau und liebevollen vierfachen Mutter und entspricht somit dem traditionellen nationalsozialistischen Frauenbild ${ }^{13}$. Die Liebesgeschichte stellt sich aber im Roman als ,Unordnung ${ }^{14}$ heraus und gefährdet vorübergehend die Effizienz der Ermittlungsinstanz, weil sie Peter Tewes von der Detektion ablenkt: „Adelaide oder der Onkel?" 15 , fragt er sich in einer schwierigen Phase der Mordaufklärung, die all seine Kräfte beanspruchen müsste.

Die Geliebte fungiert aber nicht nur als Störung, sondern auch als Helferin, denn sie trägt entscheidend zur Lösung des Falles bei. Peter Tewes verfügt außerdem über einen zweiten „Bundesgenosse[n]"16, der einen großen Beitrag zur Mordaufklärung leistet: seinen Freund Johannes Clausen. Diese Figur erfüllt eine interessante Funktion, denn sie führt die politische Ebene in den Roman ein. Die Zeit vor der Märzrevolution 1848 dient als Hintergrund des Erzählten und wird auch inhaltlich relevant, denn reale Freiheitskämpfer treten als Romanfiguren auf, denen Johannes Clausen bei der Flucht vor den reaktionären Behörden hilft. Die wichtigste Rolle unter ihnen spielt der liberale Dichter und Philologe August Heinrich Hoffmann von Fallersleben (1798-1874), der in der literarischen Fiktion des Romans von Peter Tewes irrigerweise sogar als Mörder Ito Diedrichs verdächtigt wird. Die Historisierung des Stoffes verhindert außerdem offene Bezüge auf den Nationalsozialismus, die jedenfalls in den meisten Kriminalromanen des Dritten Reiches ebenso fehlen, wie Anspielungen auf rassische und antisemitische Inhalte, NS-Mythologeme und -Symbole ${ }^{17}$.

12. - Siegfried Kracauer, „Der Detektivroman. Ein philosophisches Traktat”, S. 147, in: Siegfried Kracauer, Werke, Inka Mülder-Bach (Hrsg.): Bd. I, Frankfurt a. M., Suhrkamp, 2006, S. 107-212. Siehe auch die dritte Regel van Dines für das Verfassen von Detektivgeschichten: „Es darf keine Liebesgeschichte geben“ (S. S. van Dine, ,Zwanzig Regeln für das Schreiben von Detektivgeschichten“, S. 143, in: Jochen Vogt (Hrsg.), Der Kriminalroman, Bd. I, München, Fink, 1992, S. 143-147.

13. - Vgl. Adolf Hitler, Mein Kampf, Zwei Bände in einem Band, München, Zentralverlag der NSDAP, 1943, S. 460.

14. - Thomas Narcejac, Il romanzo poliziesco [Une machine à lire: le roman policier], Milano, Garzanti, 1975, S. 75.

15. - Georg von der Vring, Die Spur im Hafen, a.a.O., S. 133.

16. - Ebd., S. 87, 124, 149.

17. - Carsten Würmann betont, dass maximal ein Fünftel der Kriminalromane zu Hitlers Zeiten propagandistisch aufgeladen ist (Carsten Würmann, Zwischen 
Trotzdem weist Die Spur im Hafen einige Züge auf, die sich in die nationalsozialistische Weltanschauung gut integrieren, obwohl von der Vring kein Anhänger der Diktatur war. Der historische Hintergrund des Romans zählt zu den im Dritten Reich beliebtesten Phasen der deutschen Geschichte, denn die Revolution 1848 wurde von den Nationalsozialisten als „Tat- und Wirklichkeitswerdung des Volksgeistes“18 gerühmt. Eine mögliche NS-Konnotierung gekennzeichnet außerdem folgendes Zitat, das auch einen Hinweis auf Hitler enthalten könnte:

[...] dieser Traum von einem größeren Vaterland! Nie wird es so ein Deutschland geben, bei unserem zwiespältigen Nationalcharakter. Dankbar - o wie dankbar sollten wir alle dafür sein, daß uns ein kleiner Fürst regiert und allen Nörglern den Mund stopft!19

Schließlich passt die revolutionäre Jugend, die ihrem Herzen folgt und sich gegen die Väter auflehnt ${ }^{20}$, in das irrationale und kämpferische Weltbild des Dritten Reiches. Interessant ist diesbezüglich, dass auch die Tathergangsanalyse von Peter Tewes eben als „Kampf“21 angesehen wird. Andererseits könnte folgende Äußerung des jungen Juristen über die traurige Lage Deutschlands zwischen den Zeilen als Kritik am Führer verstanden werden: „In diesem Augenblick wurde es mir grausam deutlich, wohin es führte, wenn eine große und kluge Nation auf die ungerechteste und kurzsichtigste Weise regiert wurde" 22 . Außerdem könnten die verfolgten Rebellen, die ihre Heimat heimlich verlassen, als Anspielung auf die Exilanten des Dritten Reiches gelten. Die offenen inhaltlichen und sprachlichen Bezüge auf Amerika bezeugen schließlich gleichermaßen, dass der Roman vor der erzwungenen Beseitigung aller anglo-amerikanischen Spuren aus der Kriminalliteratur verfasst wurde, und dass von der Vring - im Unterschied zu den Nationalsozialisten - ein positives Bild Amerikas als Land der Freiheit und der uneingeschränkten Selbstentfaltung gibt ${ }^{23}$. Ähnlich wie sein Autor ist Die Spur

Unterhaltung und Propaganda, a.a.O., S. 273). Eine Erklärung dazu versucht Vincent Platini, der behauptet: „La loi de mai 1933 sur la protection des symboles nationaux (Gesetz zum Schutze der nationalen Symbole) empêche la récupération de l'apparat nazi par la culture de masse [...]. Trop dévalorisé pour faire œuvre de propagande, le Krimi garde des apparences apolitiques” (Vincent Platini, „Le Krimi sous le Troisième Reich: une invention de l'étranger", S. 4, in: Transatlantica. Revue d'études américaines, 2012, 1, S. 2-27).

18. - Franz Koch, Geschichte deutscher Dichtung, Hamburg, Hanseatische Verlagsanstalt, 1937, S. 187.

19. - Georg von der Vring, Die Spur im Hafen, a.a.O., S. 141.

20. - Ebd.

21. - Ebd., S. 193.

22. - Ebd., S. 141.

23. - Vgl. ebd., S. 21, 48, 153, 159, 267. 
im Hafen auf der ideologischen Ebene nicht, gleichgeschaltet', trotzdem kann man in diesem Werk eine leichte Vereinnahmung feststellen, die wahrscheinlich auch durch den Geschmack des Publikums bedingt wurde.

Genauso wie die Liebe sorgt auch die Politik für Irrwege und Verzögerungen, die die Detektion belasten, weil Peter Tewes bei der gefährlichen Flucht der Verfolgten hilft und dadurch die Fahndung nach dem Mörder gefährdet. Die angewandten Techniken der Wahrheitsfindung sind dem Dritten Reich nicht zeitgemäß, da die Handlung eben in der ersten Hälfte des 19. Jahrhunderts angesiedelt ist; außerdem spielt die in der NS-Kriminalliteratur sonst sehr moderne und leistungsfähige Polizei in Die Spur im Hafen eine sehr geringe Rolle. Die Ermittlung wird also nur von Peter Tewes mit der teilweise behindernden Hilfe seiner Geliebten und Johannes Clausen und mit geringen Detektionsmitteln geführt, bis die Kernfragen der Gattung ,who? how? why? ${ }^{24}$ zum Schluss beantwortet werden. Den Zweifeln und Unsicherheiten eines nicht immer rationalen Ermittlers ${ }^{25}$ widersprechen die Entschlossenheit und das Selbstbewusstsein der fiktiven Detektive im Nationalsozialismus, die im Unterschied zu Peter Tewes meistens einen guten Überblick über die Situation haben und sehr konkret vorgehen. Der Assessor ist in der Tat kein „Gehirn-Detektiv“26, sondern stützt sich, wie im Dritten Reich üblich, hauptsächlich auf Zeugenbeweise, die er aber oft emotional statt rational auswertet ${ }^{27}$ und die $\mathrm{zu}$ dem in NS-Kriminalromanen häufig geschilderten Geständnis der Täter führen. Die geringe Rolle der ratio zeigt sich auch auf der sprachlichen Ebene, in der der Wortschatz aus dem Bereich der Logik fehlt, obwohl oft Wörter vorkommen, die Kriminalgeschichten kennzeichnen ${ }^{28}$. Der einfache, leidenschaftliche und nicht-rhetorische Stil von der Vrings stimmt außerdem mit der von den Behörden des Dritten Reiches erwünschten formalen Tendenz der Unterhaltungsliteratur überein, die auf eine breite und leichte Rezeption zielte. Einerseits entsprechen also Inhalt und Form dieses Werkes dem typischen Detektionsschema der

24. - Peter Nusser, Der Kriminalroman, Stuttgart, Metzler, $1992^{2}$, S. 5.

25. - "Das waren lauter verschiedene Kräfte, die mich anpackten. Jede zog mich nach einer andren Seite. Welche würde siegen?", fragt sich der Assessor (Georg von der Vring, Die Spur im Hafen, a.a.O., S. 133).

26. - Klaus-Peter Walter, Reclams Krimi-Lexikon. Autoren und Werke, Stuttgart, Reclam, 2002, S. 40.

27. - "Als ich sein Stöhnen hörte, begann ich daran zu zweifeln, daß dieser Mensch der von mir gesuchte Mörder meines Onkels sein sollte. Sein Herz klagte so echt, daß es mir durch die Seele schnitt“, sagt Peter Tewes und damit streicht er einen Verdächtigen aus (Georg von der Vring, Die Spur im Hafen, a.a.O., S. 54).

28. - Z. B. „Mord“, „Beweise“, Spur, „aufklären“, „Fall“, „Beobachtungen“, „Beweisstücke“, „ermitteln“, „Lupe“ (Ebd., S. 12, 121, 125, 147, 148, 154, 179, 201). 
deutschsprachigen Kriminalliteratur der dreißiger und vierziger Jahre und seinen Darstellungsmöglichkeiten, andererseits weicht aber Die Spur im Hafen von einigen Eigenschaften der meisten NS-Kriminalromane $a b$ und bezeugt die relative Freiheit, der sich die Unterhaltungsliteratur zu Hitlers Zeiten erfreuen konnte. Sowohl die Bereicherung der Ermittlung durch eine problematische Liebesgeschichte und durch eine Freundschaftsgeschichte mit politischen Implikationen als auch die Entscheidung, die Detektion in einem historisch abenteuerlichen Kontext mit einigen Zügen der Mystery spielen zu lassen ${ }^{29}$, erzeugen eine überzeugende Kontamination unterschiedlicher Gattungen, die den Untertitel ,Roman ${ }^{6}$ - statt ,Kriminalroman ${ }^{6}$ - erklärt, die Spannung erhöht und imstande ist, eine sehr breite Leserschaft zu gewinnen.

Eine tragende Funktion erfüllen auch die autobiographischen Züge des Werkes, denn Anlass des Schreibens war eine in der Kindheit des Autors von der Großmutter erzählte reale, unaufgeklärte Mordgeschichte des 19. Jahrhunderts, die der ,Fall Hansen“ widerspiegelt ${ }^{30}$. Das künstlerische Werk erfüllt also eine einordnende Funktion, vervollkommnet die Wirklichkeit und bereichert sie, indem literarische Ermittlungspraktiken zur fiktionalen Aufdeckung eines realen Verbrechens führen. Eine enge Verbindung besteht außerdem zwischen Autor und Detektiv, nicht nur weil Peter Tewes einige Züge von der Vrings aufweist ${ }^{31}$, sondern auch weil der Ermittler zum Ich-Erzähler wird und sich im Nachwort deutlich als ,Schriftsteller ${ }^{6}$ stilisiert ${ }^{32}$. Das Verhältnis von Schreiben und Detektion wird durch Indizien (das Notizbuch Ito Diederichs und den Obduktionsbericht), durch die Arbeitsweise des Ermittlers (seine eigene Notizen im Notizbuch des Onkels) und durch die narrativen Strategien des Romans (der Ermittler als Verfasser des Kriminalromans) sichtbar. Geschriebene, gesprochene, abgelauschte, erzwungene oder gestandene Worte liegen also der Detektion und ihrer literarischen Schilderung zugrunde. Besonders treffend ist also in diesem Falle Heißenbüttels theoretische Beobachtung, dass ein Detektiv ,jemandem [gleicht],

29. - Peter hat hin und wieder den Eindruck, die Stimme des toten Onkels zu hören und seine Anwesenheit zu spüren (ebd., S. 20, 28, 192). Sogar die Entscheidung, seine Erlebnisse als Ermittler zu Papier zu bringen, wird dadurch gerechtfertigt, dass ,etwas ihn trieb', als er am Schreibtisch unter dem Porträt Ito Diedrichs saß (ebd., S. 286).

30. - Georg von der Vring, "Die Vorgeschichte zu ,Spur im Hafen““, in: Georg von der Vring, Die Spur im Hafen. Roman, München, Schneekluth, 1986, S. 249-251.

31. - Berührungspunkte ergeben sich, wenn man den Roman mit von der Vrings Leben vergleicht, das u.a. in Schlossmuseum Jever und Schifffahrtsmuseum Brake (Hrsg.), Georg von der Vring 1889-1968. Eine Ausstellung zum 100. Geburtstag des Dichters und Malers (a.a.O.) ausführlich geschildert ist.

32. - Georg von der Vring, Die Spur im Hafen, a.a.O., S. 286. 
der aus einzelnen Strichen zunächst eine Schrift und dann einen Text erschließt"33.

Barbara Korte und Sylvia Paletschek behaupten, aufgrund ihres Interesses an gesellschaftlicher Ordnung böten Kriminalromane Hinweise auf die Zeit ihrer Entstehung. Eben in dem Ordnungsbegriff liegt ein weiterer Schwerpunkt des Romans, der sich für eine mögliche NS-Vereinnahmung gut eignen könnte. In Bezug auf die Ermittlung erklärt Peter Tewes: „Ich wollte eine Pflicht erfüllen, die ich mir freiwillig auferlegt hatte, für den Onkel, für die Ordnung in der Welt, für die Gerechtigkeit"34. Die Detektion übt also in diesem Text auch eine im NS-Kriminalroman typische ,herrschaftsstabilisierende Wirkung“35 aus, die eine überindividuelle Bedeutung gewinnt, indem der Ermittler die Fahndung nach den Tätern literarisch aufarbeitet und darüber einen Roman verfasst. Personale Erlebnisse werden somit der Gemeinschaft zugänglich, und der Ermittler spielt die Rolle des „Agent[en] sozialer Gerechtigkeit", die für die innere Sicherheit des Dritten Reiches von großer Bedeutung ist. Die Spur im Hafen zeigt also sowohl in der Darstellung von Mord und Ermittlungspraktiken als auch in der Funktionalisierung des Ermittlers deutliche zeit- und gattungsbedingte Eigenschaften, die gesellschaftlichen und künstlerischen Normen des Dritten Reiches geschuldet sind, obwohl weder der Roman noch sein Autor als ,nationalsozialistisch' bezeichnet werden können.

33. - Helmut Heißenbüttel, "Spielregeln des Kriminalromans", S. 362 in: Jochen Vogt (Hrsg.), Der Kriminalroman, Bd. II, a.a.O., S. 356-371.

34. - Georg von der Vring, Die Spur im Hafen, a.a.O., S. 36.

35. - Carsten Würmann, „Deutsche Kommissare ermitteln“, a.a.O., S. 240. 
